\title{
Percutaneous endovascular biopsy in the diagnosis of venous sinus lesions: technical note
}

\author{
Tao Quan, MD,, Xinqiang Li, MD, ${ }^{2}$ Haowen Xu, MD, PhD, ${ }^{1}$ Yanan Lin, MD, ${ }^{3}$ Chao Liu, MD, ${ }^{1}$ \\ Dongdong Li, MD, ${ }^{1}$ and Sheng Guan, MD, PhD ${ }^{1}$
}

\begin{abstract}
Departments of ${ }^{1}$ Interventional Neuroradiology, ${ }^{2}$ Pathology, and ${ }^{3}$ Magnetic Resonance, The First Affiliated Hospital of Zhengzhou University, Henan, China
\end{abstract}

\begin{abstract}
Intracranial hypertension $(\mathrm{IH})$ may be misdiagnosed owing to the lack of typical imaging features and pathological confirmation of the sinus lesions. The authors report the use of percutaneous endovascular biopsy (PEB) for the diagnosis of $\mathrm{IH}$ in patients with venous sinus lesions. A total of 9 patients (age $46 \pm 9$ years) underwent PEB between June 2016 and August 2017. All patients underwent lumbar puncture and contrast-enhanced MRI before the procedure. PEB was technically successful in 6 patients. No intra- or postprocedural complications occurred. The confirmed lesions were meningioma in 2 patients and fibrous thrombus in 4 patients. All patients received individualized treatment. PEB of venous sinus lesions is a safe and efficient method to establish an early diagnosis and appropriate treatment in patients with $\mathrm{IH}$.
\end{abstract}

https://thejns.org/doi/abs/10.3171/2018.3.JNS173143

KEYWORDS intracranial hypertension; angiography; endovascular biopsy; pathology; vascular disorders; diagnostic technique

I NTRACRANIAL hypertension (IH) is a common clinical issue in neurology and neurosurgery. The pathognomonic signs consist of headache, vomiting, and papilledema. ${ }^{10} \mathrm{IH}$ may be caused by obstruction of venous drainage..$^{21,23}$ The most frequent causes of obstruction are venous sinus lesions, including acute and chronic thrombosis; damage or ligation after trauma; meningeal inflammation; metabolic disturbances; meningeal metastases; and benign tumors. ${ }^{21}$ Some studies have demonstrated that the stenosis of sinuses is associated with idiopathic intracranial hypertension (IIH). ${ }^{3-5,7,18,19}$ However, the abnormal imaging findings in IIH are nonspecific and can also be found in patients with raised intracranial pressure (ICP) due to cerebral venous thrombosis. ${ }^{5,12,18}$ Compression caused by a small lesion or invasion of the venous sinus may lead to stenosis of the local region..$^{15}$ Despite major improvements in MRI examination over the past decade, misinterpretation of brain MRI and/or CT findings may lead to a delayed diagnosis in patients presenting with raised ICP..$^{18}$ Results of noninvasive imaging techniques are often unclear with regard to identification of the underlying pathology, and the definitive diagnosis is frequently determined during surgery. ${ }^{17}$ In some patients, small ve- nous sinus lesions may be misdiagnosed as IIH due to the absence of imaging findings. Consequently, a majority of these patients receive conventional medical therapy for several months with an unfavorable clinical outcome..$^{20,23,24}$ Therefore, early diagnosis of sinus lesions with pathological confirmation is crucial for an effective treatment plan. Owing to the complex anatomy associated with venous sinus lesions, pathological examination is challenging if it cannot be performed during surgical resection.

In this study, we investigated a new method using percutaneous endovascular biopsy (PEB) for diagnosis in IH patients with venous sinus lesions. Patient clinical profiles, procedure technical success and safety, and clinical success were evaluated. On the basis of a comprehensive literature search, we believe that this is the first patient cohort in which the feasibility and safety of this method has been demonstrated.

\section{Methods}

From June 2016 to August 2017, 9 patients with IH underwent PEB for confirmation of the presence of an intrasinus lesion and assessment of its anatomical relationship

ABBREVIATIONS DAVF = dural arteriovenous fistula; ICP = intracranial pressure; $\mathrm{IH}=$ intracranial hypertension; $\mathrm{IIH}$ = idiopathic $\mathrm{IH}$; PEB = percutaneous endovascular biopsy; MRBTI = magnetic resonance black-blood thrombus imaging; MRV = magnetic resonance venography.

SUBMITTED December 15, 2017. ACCEPTED March 19, 2018.

INCLUDE WHEN CITING Published online August 24, 2018; DOI: 10.3171/2018.3.JNS173143. 
with nearby structures. In all 9 patients, diagnosis of acute intracranial venous sinus thrombosis was excluded by use of a contrast-enhanced magnetic resonance black-blood thrombus imaging (MRBTI) technique before the procedure. ${ }^{25}$

All patients provided informed consent for PEB after being informed about the procedure and its benefits and potential complications. All procedures performed in the studies involving human participants were in accordance with the ethical standards of the institutional committee

\section{Description of Technique}

All patients were placed in the supine position under local anesthesia with full heparinization and neurological monitoring. Screening coagulation tests were ordered routinely. Both femoral arterial and venous access were obtained in all 9 patients. Consequently, manual compression at the site of the vascular puncture was performed over a period of 10 minutes in order to achieve hemostasis. In 6 patients, a 70-cm 8-Fr vascular introducer sheath (Cook) was used to provide better support. In the other 3 patients, an $11-\mathrm{cm} 8-\mathrm{Fr}$ vascular introducer sheath (Cordis) was used. Before PEB, the lesion in the venous sinus was confirmed with an angiogram. ICP manometry was performed with a PROGREAT microcatheter (Terumo) attached to the pressure transducer. An 8-Fr ENVOY guiding catheter (Codman) and a 6-Fr naive catheter (ev3) were cautiously positioned proximal to the lesion in 8 patients. A 1.8-mm biopsy forceps device (Micro-Tech) was gently advanced upward with both stainless-steel hinged cutting jaws in the closed position. The biopsy device was advanced under fluoroscopic guidance. In 1 patient, a 2.3$\mathrm{mm}$ biopsy forceps device (Micro-Tech) was gently advanced in a 70-cm 8-Fr vascular introducer sheath, which was placed in the left jugular foramen. An introducer tip with hinged jaws was guided in the open position and gently placed into the lesion, anchoring the jaws in the lesion, and then the hinged jaws were maneuvered into the closed position. The naive catheter was pushed to a fixed position, followed by withdrawal of the biopsy forceps device. Two to 4 tissue samples of the lesion were obtained. For the $1.8-\mathrm{mm}$ device the theoretical specimen volume was 5 $\mathrm{mm}^{3}$. However, in some cases the specimen obtained was larger than the volume of the jaws. After the operation, we used protamine to reverse the effects of heparin. The specimens were fixed in both normal saline and formalin solutions and evaluated pathologically.

\section{Results}

A cohort of 9 patients, comprising 3 men and 6 women with a mean \pm SD age of $46 \pm 9$ years, was reviewed. A summary of patient characteristics and pathological results is presented in Table 1. Four patients presented with headache and 4 patients presented with papilledema. One patient presented with both headache and papilledema. Lumbar puncture analysis results showed high CSF pressure in all 9 patients (range $330-510 \mathrm{~mm} \mathrm{H}_{2} \mathrm{O}$ ). Five intrasinus lesions were located in the transverse sinus, 3 in the sigmoid sinus, and 1 in the jugular foramen. One patient (case 9) presented with left sigmoid sinus stenosis complicated by dural arteriovenous fistula (DAVF).

Technical success in obtaining a biopsy specimen was achieved in $6 / 9$ patients $(66.7 \%)$. A total of 7 intravascular lesions were observed and evaluated, 1 lesion in each of 5 patients and 2 lesions in 1 patient. A mean of 3 samples was obtained per biopsy procedure (range 2-5). Some patients experienced a transient headache and recovered completely after the procedure. No intra- or postprocedural complications occurred. The confirmed lesions were meningioma in 2 patients and fibrous thrombus in 4 patients. In one patient (case 1), a fibrous thrombus was observed in the first procedure and meningioma was confirmed in the second attempt. Five patients underwent venous sinus stenting, 1 patient underwent tumor resection, 1 patient received a ventriculoperitoneal shunt, 1 patient received anticoagulation therapy, and 1 patient underwent embolization of DAVF.

\section{Illustrative Cases \\ Case 1}

A 37-year-old woman had a 3-month history of progressive visual deterioration. The fundus examination exhibited bilateral stage 2 papilledema. The lumbar puncture showed high CSF pressure (340-360 $\left.\mathrm{mm} \mathrm{H}_{2} \mathrm{O}\right)$. Enhanced MR images revealed a lesion located in the left

TABLE 1. Summary of patient characteristics and pathological results

\begin{tabular}{|c|c|c|c|c|c|c|c|c|}
\hline $\begin{array}{l}\text { Case } \\
\text { No. }\end{array}$ & Sex & $\begin{array}{l}\text { Age } \\
\text { (yrs) }\end{array}$ & Presentation & $\begin{array}{c}\mathrm{ICP} \\
\left(\mathrm{mm} \mathrm{H}_{2} \mathrm{O}\right)\end{array}$ & Lesion Location & $\begin{array}{l}\text { Lesion } \\
\text { Type }\end{array}$ & Pathology & Treatment \\
\hline 1 & $\mathrm{~F}$ & 46 & Papilledema & 337 & Right transverse sinus & Stenosis & - & Stenting \\
\hline 2 & M & 50 & Papilledema & 372 & Left sigmoid sinus & Stenosis & Fibrous thrombus & Stenting \\
\hline 3 & $\mathrm{~F}$ & 37 & Papilledema & 354 & Left jugular foramen & Occlusion & $\begin{array}{l}\text { Fibrous thrombus (1st PEB); } \\
\text { meningioma (2nd PEB) }\end{array}$ & CSF shunting \\
\hline 4 & $\mathrm{~F}$ & 66 & Headache & 350 & Left transverse sinus & Occlusion & Meningioma & Resection \\
\hline 5 & M & 38 & Papilledema & 516 & Right transverse sinus & Stenosis & - & Stenting \\
\hline 6 & $\mathrm{~F}$ & 40 & Papilledema & 502 & Right transverse sinus & Stenosis & Fibrous thrombus & Stenting \\
\hline 7 & $\mathrm{~F}$ & 50 & Headache, vomiting & 385 & Left sigmoid sinus & Stenosis & Fibrous thrombus & Anticoagulation \\
\hline 8 & $\mathrm{~F}$ & 42 & Headache, papilledema & 402 & Right transverse sinus & Stenosis & - & Stenting \\
\hline 9 & $M$ & 46 & Headache & 358 & Left sigmoid sinus & Stenosis & Fibrous thrombus & DAVF embolization \\
\hline
\end{tabular}



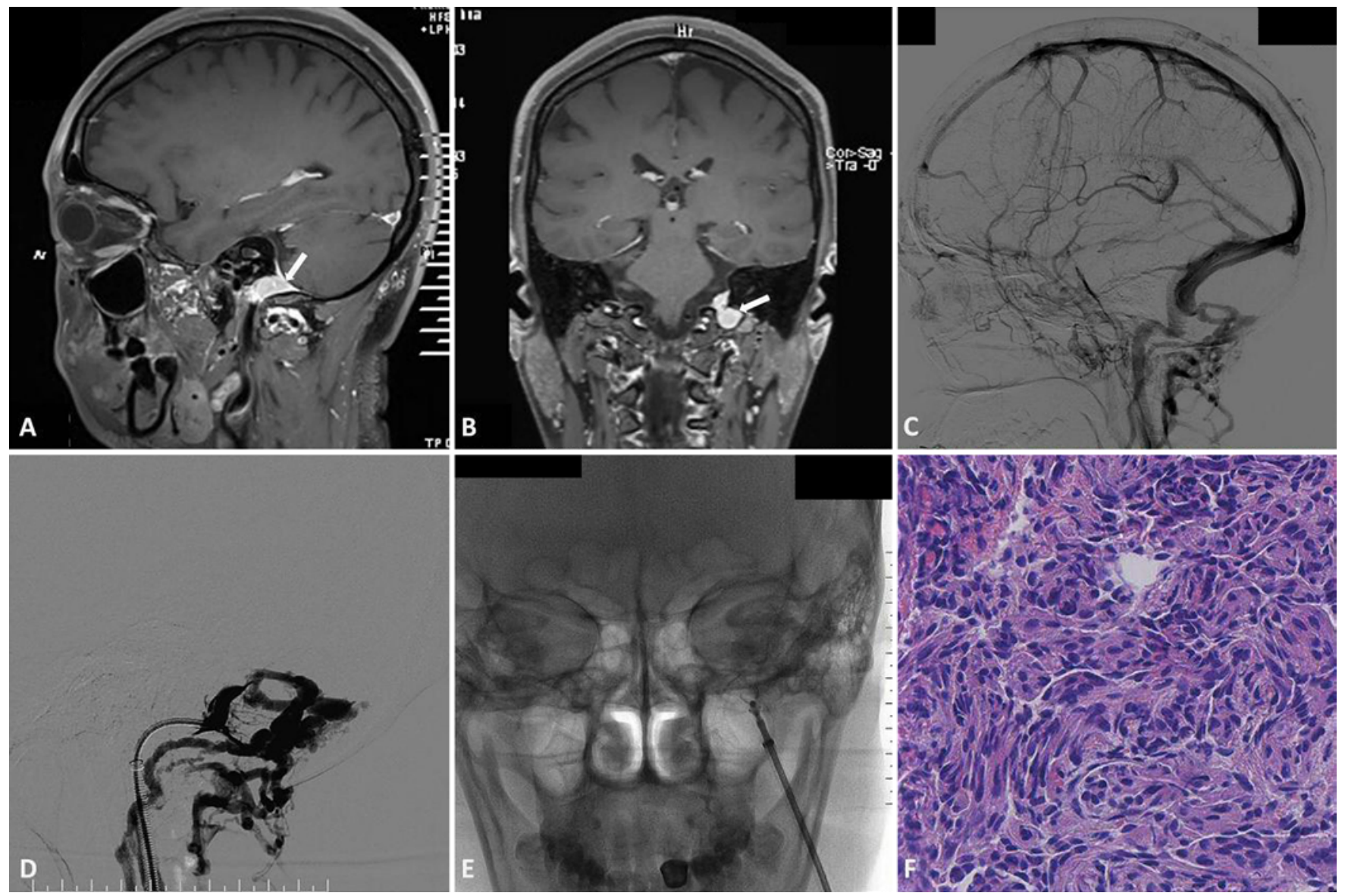

FIG. 1. Case 1. A and B: Enhanced MR images demonstrating the lesion (white arrows) located in the left jugular foramen. $\mathbf{C}$ and D: Angiographic images demonstrating occlusion of the left sigmoid sinus and the collateral veins. E: Image of a 2.3-mm biopsy forceps device used to obtain a specimen. F: Pathological analysis of the biopsy specimen revealed meningioma (H \& E; magnification $\times 400)$.

jugular foramen (Fig. 1A and B). A digital subtraction angiogram revealed an occlusion of the left sigmoid sinus (Fig. 1C and D). The PROGREAT microcatheter recorded a mean pressure of $354 \mathrm{~mm} \mathrm{H}_{2} \mathrm{O}$ in the confluence of the sinus. During the patient's first procedure, a 1.8-mm biopsy forceps device was advanced upward in an 8-Fr ENVOY guiding catheter that was placed in the left jugular foramen. Five samples were obtained, and pathological analysis revealed a thrombus. After 8 months of oral anticoagulant therapy, the patient's symptoms did not improve significantly and she received further treatment. At this time a second enhanced MR image showed that the lesion was not changed. In the second procedure after 8 months, a 2.3-mm biopsy forceps device was gently advanced upward in a 70-cm 8 -Fr vascular introducer sheath that was placed in the left jugular foramen (Fig. 1E). Three samples were obtained, and pathological analysis revealed meningioma (Fig. 1F). This patient underwent a CSF diversion procedure, and the papilledema had improved at the 3-month follow-up examination.

\section{Case 2}

A 40-year-old woman had a 50-day history of progressive visual deterioration. Bilateral papilledema was con- firmed by fundus photography. Lumbar puncture showed a high CSF pressure $\left(500 \mathrm{~mm} \mathrm{H}_{2} \mathrm{O}\right)$. With the use of magnetic resonance venography (MRV), this patient was initially diagnosed as having IIH with right transverse sinus stenosis (Fig. 2A). Subsequently, an enhanced MR image revealed a small lesion located in the right transverse sinus (Fig. 2B). A digital subtraction angiogram revealed stenosis of the right transverse sinus (Fig. 2C). The PROGREAT microcatheter recorded a mean pressure of $502 \mathrm{~mm} \mathrm{H}_{2} \mathrm{O}$ in the superior sagittal sinus. A 1.8-mm biopsy forceps device was gently advanced upward in an 8-Fr ENVOY guiding catheter that was placed in the right sigmoid sinus (Fig. 2D). Three samples were obtained and fixed in formalin solutions (Fig. 2E and F). This patient underwent stent placement (Fig. 2G). Pathological analysis revealed a fibrous thrombus (Fig. $2 \mathrm{H}$ ). Lumbar puncture showed low CSF pressure (90-100 $\mathrm{mm} \mathrm{H}_{2} \mathrm{O}$ ) 3 days after the procedure. The papilledema had improved after 6 months of follow-up.

\section{Discussion}

IH caused by venous sinus stenosis or occlusion has been studied for decades. ${ }^{6,9,21,24}$ Any lesion that obstructs 

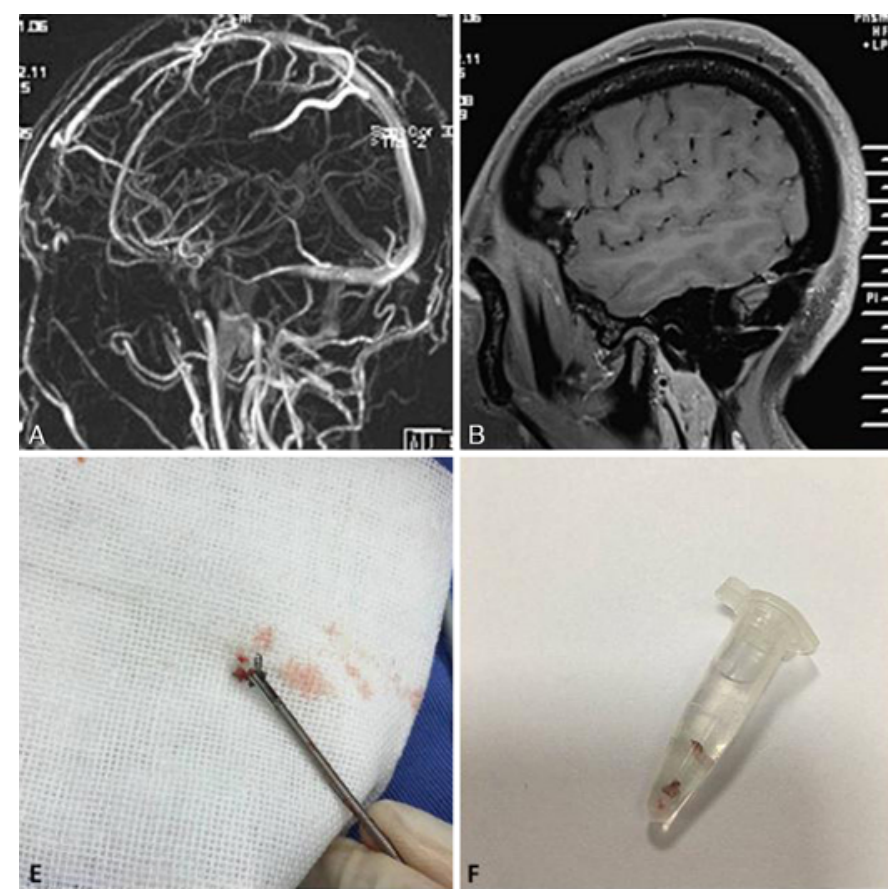
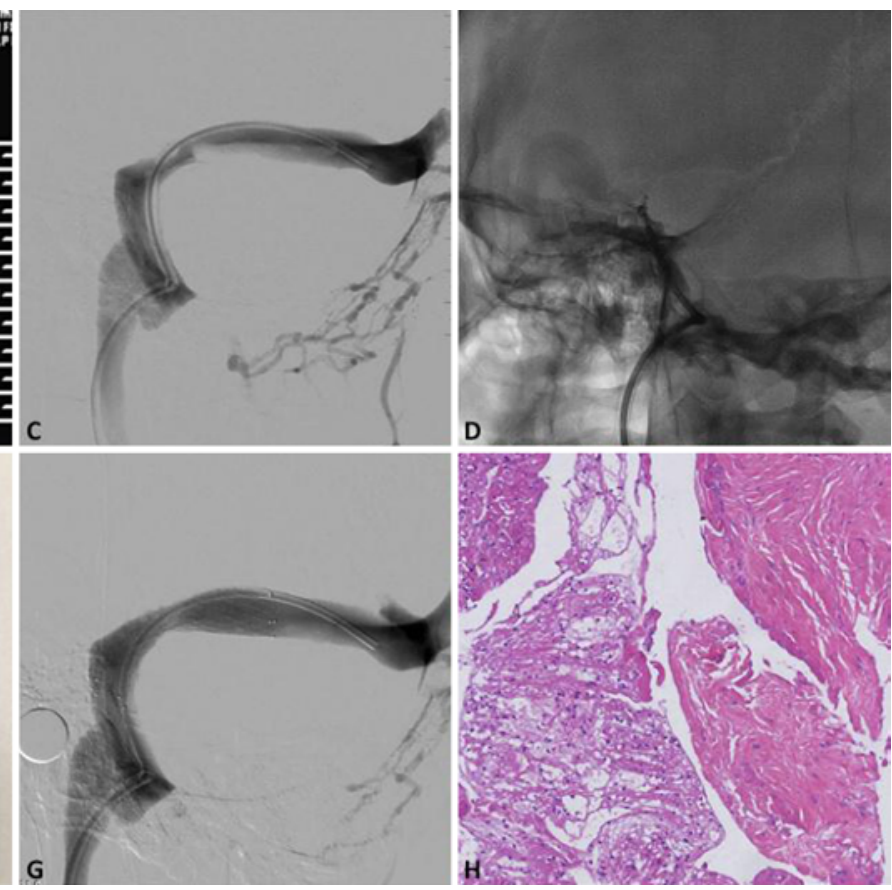

FIG. 2. Case 2. MRV (A) and angiogram (C) showing right transverse sinus stenosis, enhanced MR image (B) showing the lesion (white arrow), and angiogram obtained after stenting (G). A 1.8-mm biopsy forceps device (D) was used to obtain a specimen (E and F), for which pathological analysis revealed a fibrous thrombus ( $\mathrm{H} \& \mathrm{E}$; magnification $\times 400)(\mathrm{H})$.

the venous pathway from the granulations to the right heart is a potential biological cause of $\mathrm{IH} .{ }^{24}$ With recent progress in imaging technology, we have been able to use the MRBTI technique ${ }^{25}$ to find small intrasinus lesions in some patients with IH in whom it was difficult to diagnose and distinguish the lesion from the patient's clinical history, especially in patients with atypical symptoms. These patients are at risk for being misdiagnosed with $\mathrm{IIH} .{ }^{8}$ In such cases, if the pathological results can be obtained accurately, subsequent treatment can be guided more effectively. However, obtaining the pathological specimens of sinus lesions using a minimally invasive approach is challenging.

Specimens from patients with hypertrophic pachymeningitis or meningioma can be obtained by standard meningeal biopsy or resection. ${ }^{13,16,22}$ However, this procedure may cause relatively severe surgical trauma. Thrombus specimens from patients with cerebral venous sinus thrombosis can be obtained by mechanical thrombectomy. ${ }^{11,14}$ PEB has been described as "a safe and efficient procedure providing the most effective technique to obtain a tissue sample of high diagnostic quality," and can be used for early diagnosis in patients with nonspecific lesions. ${ }^{17}$ To our knowledge, no previous studies have demonstrated the feasibility of PEB for early diagnosis in patients with venous sinus lesions.

Stenosis of the sinuses appears as smooth narrowing of the venous sinus (extraluminal type) or an abrupt filling defect (intraluminal type). ${ }^{5}$ In the current cohort, the angiographic images of 7 stenosis cases demonstrated the intraluminal type and were in accordance with the results of enhanced MRI. Specimens from 3 patients revealed fibrous thrombus, which is the putative cause of venous sinus stenosis and a secondary cause of $\mathrm{IH}$. We speculated that some IIH patients experienced venous sinus thrombosis, without onset of acute symptoms, and residual fibrous thrombosis caused chronic obstruction of the venous sinus.

In recent years, transvenous dural venous sinus stent (DVSS) insertion has emerged as a potential therapy for resistant IH cases. More than $20 \%$ of DVSS patients developed restenosis in their medium- or long-term follow-up. ${ }^{1,2}$ It is currently unclear which types of lesions are prone to restenosis. If these lesions can be identified by using biopsy specimens, the surgeon may tailor treatment, such as shunting, according to the patient's pathological result.

Technically, assisted by the guiding or naive catheter, the PEB device exhibited the advantages of being flexible and able to follow even the most tortuous vascular paths up to the lesions. Fluoroscopic imaging enabled us to evaluate the precise position of the biopsy device in real time to avoid sampling a nontargeted biopsy site, particularly the normal vessel wall. In order to achieve a biopsy material of optimal quality, the introducer sheath required positioning toward the surface of the lesion, which was stabilized when the biopsy device was advanced to the target lesion. Owing to the rigidity of the device, the ideal positioning was not always feasible, especially in the lesser curvature of the sigmoid sinus where the trajectory of the introducer included multiple curvatures. Because of the unsatisfactory flexibility of the head, lesions at the lower and inner wall were challenging to obtain compared to lesions of the upper and lateral wall. The lesions of 3 patients could not be located at the lower wall of the transverse sinus or inner wall of the sigmoid sinus. 
Because of the rarity of this disease, one major limitation of the study is the small number of enrolled patients. This technique may be improved for use in additional procedures for which a flexible type of biopsy forceps device is essential.

\section{Conclusions}

Percutaneous endovascular biopsy of venous sinus lesions is a safe and efficient alternative procedure. This technique may help the surgeons to obtain an early diagnosis and choose appropriate treatment in patients with $\mathrm{IH}$.

\section{References}

1. Aguilar-Pérez M, Martinez-Moreno R, Kurre W, Wendl C, Bäzner H, Ganslandt O, et al: Endovascular treatment of idiopathic intracranial hypertension: retrospective analysis of immediate and long-term results in 51 patients. Neuroradiology 59:277-287, 2017

2. Asif H, Craven CL, Siddiqui AH, Shah SN, Matloob SA, Thorne L, et al: Idiopathic intracranial hypertension: 120-day clinical, radiological, and manometric outcomes after stent insertion into the dural venous sinus. J Neurosurg [epub ahead of print October 6, 2017; DOI: 10.3171/2017.4.JNS162871]

3. Avery MB, Sambrano S, Khader Eliyas J, Eesa M, Mitha AP: Accuracy and precision of venous pressure measurements of endovascular microcatheters in the setting of dural venous sinus stenosis. J Neurointerv Surg 10:387-391, 2018

4. Baykan B, Ekizoğlu E, Altıkka Uzun G: An update on the pathophysiology of idiopathic intracranial hypertension alias pseudotumor cerebri. Agri 27:63-72, 2015

5. Bidot S, Saindane AM, Peragallo JH, Bruce BB, Newman NJ, Biousse V: Brain imaging in idiopathic intracranial hypertension. J Neuroophthalmol 35:400-411, 2015

6. Damak M, Crassard I, Wolff V, Bousser MG: Isolated lateral sinus thrombosis: a series of 62 patients. Stroke 40:476-481, 2009

7. Esfahani DR, Stevenson M, Moss HE, Amin-Hanjani S, Aletich V, Jain S, et al: Quantitative magnetic resonance venography is correlated with intravenous pressures before and after venous sinus stenting: implications for treatment and monitoring. Neurosurgery 77:254-260, 2015

8. Fisayo A, Bruce BB, Newman NJ, Biousse V: Overdiagnosis of idiopathic intracranial hypertension. Neurology 86:341350,2016

9. Hartel M, Kluczewska E, Gancarczyk-Urlik E, Pierzchała $\mathrm{K}$, Bień K, Zastawnik A: Cerebral venous sinus thrombosis. Phlebology 30:3-10, 2015

10. Iencean SM, Poeata I, Iencean AS, Tascu A: Cerebral venous etiology of intracranial hypertension and differentiation from idiopathic intracranial hypertension. Kaohsiung J Med Sci 31:156-162, 2015

11. Ilyas A, Chen CJ, Raper DM, Ding D, Buell T, Mastorakos P, et al: Endovascular mechanical thrombectomy for cerebral venous sinus thrombosis: a systematic review. J Neurointerv Surg 9:1086-1092, 2017

12. Julayanont P, Karukote A, Ruthirago D, Panikkath D, Panikkath R: Idiopathic intracranial hypertension: ongoing clinical challenges and future prospects. J Pain Res 9:87-99, 2016

13. Kupersmith MJ, Martin V, Heller G, Shah A, Mitnick HJ: Idiopathic hypertrophic pachymeningitis. Neurology 62:686-694, 2004
14. Li G, Zeng X, Hussain M, Meng R, Liu Y, Yuan K, et al: Safety and validity of mechanical thrombectomy and thrombolysis on severe cerebral venous sinus thrombosis. Neurosurgery 72:730-738, 2013

15. Mariniello G, Giamundo A, Donzelli R, Severino R, Russo $\mathrm{C}$, Elefante A, et al: Intracranial hypertension due to meningioma of the unique transverse sinus. Neuroradiol J 26:209-212, 2013

16. Mazur MD, Cutler A, Couldwell WT, Taussky P: Management of meningiomas involving the transverse or sigmoid sinus. Neurosurg Focus 35(6):E9, 2013

17. Pomoni A, Sotiriadis C, Gay F, Jouannic AM, Qanadli SD: Percutaneous endovascular biopsy of intravascular masses: efficacy and safety in establishing pre-therapy diagnosis. Eur Radiol 28:301-307, 2018

18. Ridha MA, Saindane AM, Bruce BB, Riggeal BD, Kelly LP, Newman NJ, et al: MRI findings of elevated intracranial pressure in cerebral venous thrombosis versus idiopathic intracranial hypertension with transverse sinus stenosis. Neuroophthalmology 37:1-6, 2013

19. Riggeal BD, Bruce BB, Saindane AM, Ridha MA, Kelly LP, Newman NJ, et al: Clinical course of idiopathic intracranial hypertension with transverse sinus stenosis. Neurology 80:289-295, 2013

20. Satti SR, Leishangthem L, Chaudry MI: Meta-analysis of CSF diversion procedures and dural venous sinus stenting in the setting of medically refractory idiopathic intracranial hypertension. AJNR Am J Neuroradiol 36:1899-1904, 2015

21. Sindou M, Auque J, Jouanneau E: Neurosurgery and the intracranial venous system. Acta Neurochir Suppl 94:167175,2005

22. Tang J, Zhang L, Zhang J, Wu Z, Xiao X, Zhou D, et al: Microsurgical management of primary jugular foramen meningiomas: a series of 22 cases and review of the literature. Neurosurg Rev 39:671-683, 2016

23. Teleb MS, Cziep ME, Lazzaro MA, Gheith A, Asif K, Remler B, et al: Idiopathic intracranial hypertension. A systematic analysis of transverse sinus stenting. Intervent Neurol 2:132-143, 2013

24. Wall M: Idiopathic intracranial hypertension. Neurol Clin 28:593-617, 2010

25. Yang Q, Duan J, Fan Z, Qu X, Xie Y, Nguyen C, et al: Early detection and quantification of cerebral venous thrombosis by magnetic resonance black-blood thrombus imaging. Stroke 47:404-409, 2016

\section{Disclosures}

The authors report no conflict of interest concerning the materials or methods used in this study or the findings specified in this paper.

\section{Author Contributions}

Conception and design: Quan. Acquisition of data: Quan. Analysis and interpretation of data: Quan. Drafting the article: Quan. Critically revising the article: Xu. Reviewed submitted version of manuscript: Quan. Administrative/technical/material support: Quan, X Li, Xu, Lin, Liu, D Li. Study supervision: Guan.

\section{Correspondence}

Sheng Guan: The First Affiliated Hospital of Zhengzhou University, Henan, China. gsradio@126.com. 